\title{
Hybrid fast multipole method applied to beam-beam collisions in the strong-strong regime
}

\author{
W. Herr and M. P. Zorzano* \\ CERN, SL Division, 1211 Geneva 23, Switzerland \\ F. Jones \\ TRIUMF, 4004 Wesbrook Mall, Vancouver, Canada V6T 2 A3
}

(Received 9 April 2001; published 30 May 2001)

\begin{abstract}
The strong-strong interactions of two colliding beams are simulated by tracking the motion of a set of macroparticles. The field generated by each distribution is evaluated using the fast multipole method together with some elements of particle-mesh methods. This technique allows us to check the exact frequencies of the coherent modes and the frequencies of oscillations of individual particles in the beam. The agreement between the simulations and analytical calculations is largely improved. Furthermore, it is an efficient method to study the coherent modes in the case of separated beams.
\end{abstract}

DOI: $10.1103 /$ PhysRevSTAB.4.054402

PACS numbers: 29.27.Bd, 29.27.Fh, 41.85.Ew

\section{INTRODUCTION}

Two colliding beams exert a force on each other which is defocusing for beams of equal polarity as in the case of the Large Hadron Collider (LHC). Solutions of the linearized Vlasov equation show that for round beams and in the case of one bunch per beam with equal parameters (intensity, beam size, betatron tune) two coherent dipole modes of oscillations appear: the $\sigma$ mode, whose frequency is equal to the unperturbed betatron tune, and the $\pi$ mode with a tune shift of $Y=1.21$, where $Y$ is the Yokoya factor [1], times the beam-beam parameter $\xi$.

In this paper the transverse coherent motion of two colliding proton beams is studied by multiparticle tracking. In a self-consistent model of the coherent interaction, the distributions of both beams evolve as a consequence of the mutual interaction and are used at the interaction points (IP) to calculate the force on the individual particles. A number of studies have been done for LHC using the so-called "soft Gaussian model" [2]. This model assumes the force experienced by a particle when traversing the counterrotating beam as originating from a Gaussian beam distribution with variable barycenters and rms beam sizes. This allows the use of an analytical expression for the forces. This Gaussian model cannot take into account the non-Gaussian deformations of the distribution and as a result underestimates the force and yields a Yokoya factor that is slightly smaller ( $Y=1.1$ in our case). This symptom has also been recently discussed by Yokoya [3]. In the worst case, this simplification can inhibit the appearance of coherent effects. Nonetheless, the use of the analytical expression of the force generated by a Gaussian beam allows simulations in a reasonable computing time and it is therefore more convenient for studies with multiple bunches.

*Present address: BULL, Paseo Doce Estrellas 2, 28042 Madrid, Spain.
It was predicted $[4,5]$ that the coherent $\pi$ mode may not be Landau damped for certain strong-strong conditions and therefore an accurate knowledge of the Yokoya factor is highly desirable.

\section{SIMULATIONS BEYOND THE SOFT GAUSSIAN MODEL}

To avoid this problem and to increase the accuracy of the simulations, we have to introduce a field solver for an arbitrary distribution of charges in space. The choice of the solver is constrained by the problems under investigation: (i) large number of particles in simulation $\left(10^{4}\right)$ and (ii) separated beams (separation 10 times the beam size or more).

A direct integration of forces (particle-particle methods) is ruled out since the necessary time grows with the square of the number of particles $\left[O\left(N_{p}^{2}\right)\right]$. For the number of particles used in our simulation this is impossible. Other possible solvers employ so-called particle-mesh methods and have been shown to give good results [6]. Their advantage is speed since the number of computations is smaller and depends on the number of grid points $N_{g}:\left[O\left(N_{g} \ln N_{g}\right)\right]$. A strong disadvantage is that particle-mesh methods have problems handling nonuniform distributions. For the case of separated beams (as in our case with the important effect of long-range collisions), most of the space is basically empty. Moving or adaptive grids may be used for that purpose, but may lead to a rather complicated structure.

Another possibility is to use fast multipole methods (FMM). In this algorithm the potential or force acting on a particle is divided into two components. The component of close particles is computed directly, and between distant particles the potential is approximated by multipole expansion $[7,8]$. This method is therefore well adapted to handle problems such as separated beams. Problems with FMM are close encounters and "charge overloading," i.e., 
for the LHC bunches, $10^{11}$ particles are represented by $10^{4}$ macroparticles.

\section{BASIC HFMM ALGORITHM}

For our problem we studied a modified version of FMM, a hybrid FMM (HFMM) [9]. It resembles a particle-mesh method for the handling of charges and superparticles; however, the forces on the superparticles are evaluated using the FMM. Smoothing can help to avoid charge overloading. The HFMM is a robust implementation of a FMM field solver, which is designed to solve the field for an arbitrary collection of discrete charges. It divides the solution domain into a grid and a halo area. The grid area is subdivided into a hierarchical tree of square regions. In the first step of the calculation, the macroparticles inside the grid are assigned to grid points. All macroparticles outside the grid are treated as discrete, independent superparticles and form the halo. The charge assignment can be done with a "nearest-grid-point" method; i.e., the charge is assigned to the nearest grid point. This is the simplest method; however, the field values are not continuous and the results are more noisy. Alternatively one can use the cloud-in-cell (CIC) charge assignment where the charge is shared between the neighboring grid points. This method gives continuous field values but requires more bookkeeping.

Finally, multipole expansions of the field are computed for every point, i.e., for each grid point as well as for every halo particle, and the program derives the resulting forces on the particles of the counterrotating beam. In the case of a CIC charge assignment, appropriate interpolation between the fields calculated for the grid points have to be applied. The grid size and shape do not have to follow any special geometry and can be chosen freely to achieve the desired speed and precision, depending on the problems under investigation. Unlike other Poisson solvers, the grid

$$
\left(\begin{array}{c}
x(n+1) \\
v_{x}(n+1)
\end{array}\right)=\left(\begin{array}{c}
\cos \left(2 \pi Q_{x}\right) \\
-\sin \left(2 \pi Q_{x}\right)
\end{array}\right.
$$

An equivalent map is applied in the vertical plane $\left(y, v_{y}\right)$.

The horizontal deflection experienced at the interaction point is

$$
\Delta v_{x}(n)=\frac{r_{p} N^{*}}{\gamma} E_{x}(x, y),
$$

where $E_{x}(x, y)$ is the horizontal force evaluated with the HFMM technique at the particle position $(x, y)$. The number of particles in the opposing beam is $N^{*}$.

For the simulation of parasitic (long-range) collisions, the same model is employed. The two beams collide with a horizontal separation $L_{x}$ (in units of $\sigma_{x}$ ). For low $\beta$ insertions we have about $90^{\circ}$ phase advance between the IP and the long-range collision region. Since in the LHC the betatron phase advance between long-range collisions points with no charges assigned are left out of the computation and the number of computations scales roughly with the number of particles. More details of the method used in this report are found in [9]. This method is already implemented in the ACCSIM program [10] to study space charge problems.

In this work we implemented the HFMM in our beambeam simulation program to evaluate the force on a test particle generated by an arbitrary charge distribution. This will be applied to study the strong-strong collision of two bunches colliding at one IP. We will study the coherent modes that are excited in the collision of two equal round bunches similar to those of LHC, when colliding head-on or separated by a constant offset at one interaction point (long-range interactions). This will enable us to obtain the correct Yokoya factor by multiparticle tracking and, in a later stage, to study in detail the modes excited by long-range interactions. Finally, it should allow us to study the possible emittance growth of collisions of partially overlapping bunches [11].

\section{TRACKING WITH HFMM}

We simulate the collision of two strong proton beams. Our variables are horizontal position $x$, vertical position $y$, horizontal angle $v_{x}=x^{\prime}$, and vertical angle $v_{y}=y^{\prime}$. The prime denotes the derivative with respect to longitudinal position $s$; e.g., $x^{\prime}$ is the slope of the horizontal trajectory.

Each of the beams has one bunch that is represented by a set of $N_{p}$ macroparticles, whose trajectories are followed over $n$ turns, assuming linear betatron motion without coupling and a beam-beam collision at one IP. At the IP every particle in the bunch experiences a deflection by the field of the counterrotating beam that depends on its position.

The deflection applied to a single particle in one of the beams is calculated using the HFMM.

The linear map from one IP to the next is

$\left.\begin{array}{l}\sin \left(2 \pi Q_{x}\right) \\ \cos \left(2 \pi Q_{x}\right)\end{array}\right)\left(\begin{array}{c}x(n) \\ v_{x}(n)+\Delta v_{x}(n)\end{array}\right)$.

on one side of the interaction region is very small, we can lump all $n_{\text {par }}$ parasitic collisions into a single collision to reduce the computing time. This overestimates the effect slightly because the bunches oscillate with different phases with respect to each other.

Because a static dipole kick would change the closed orbit of the bunch, the static kick from the long-range collision must be subtracted [12]. The beam-beam longrange kick used in our simulation code is then

$$
\begin{aligned}
\Delta v_{x}(n)= & n_{\mathrm{par}} \frac{2 r_{p} N_{p}^{*}}{\gamma} \\
& \times\left[E_{x}\left(x+L_{x} \sigma_{x}, y\right)-D_{x}\left(L_{x} \sigma_{x}, 0\right)\right],
\end{aligned}
$$

where $D_{x}\left(L_{x} \sigma_{x}, 0\right)=-1 / L_{x} \sigma_{x}\left[1.0-\exp \left(-\frac{L_{x}^{2}}{2.0}\right)\right]$ is the (constant) dipole kick generated by a Gaussian 

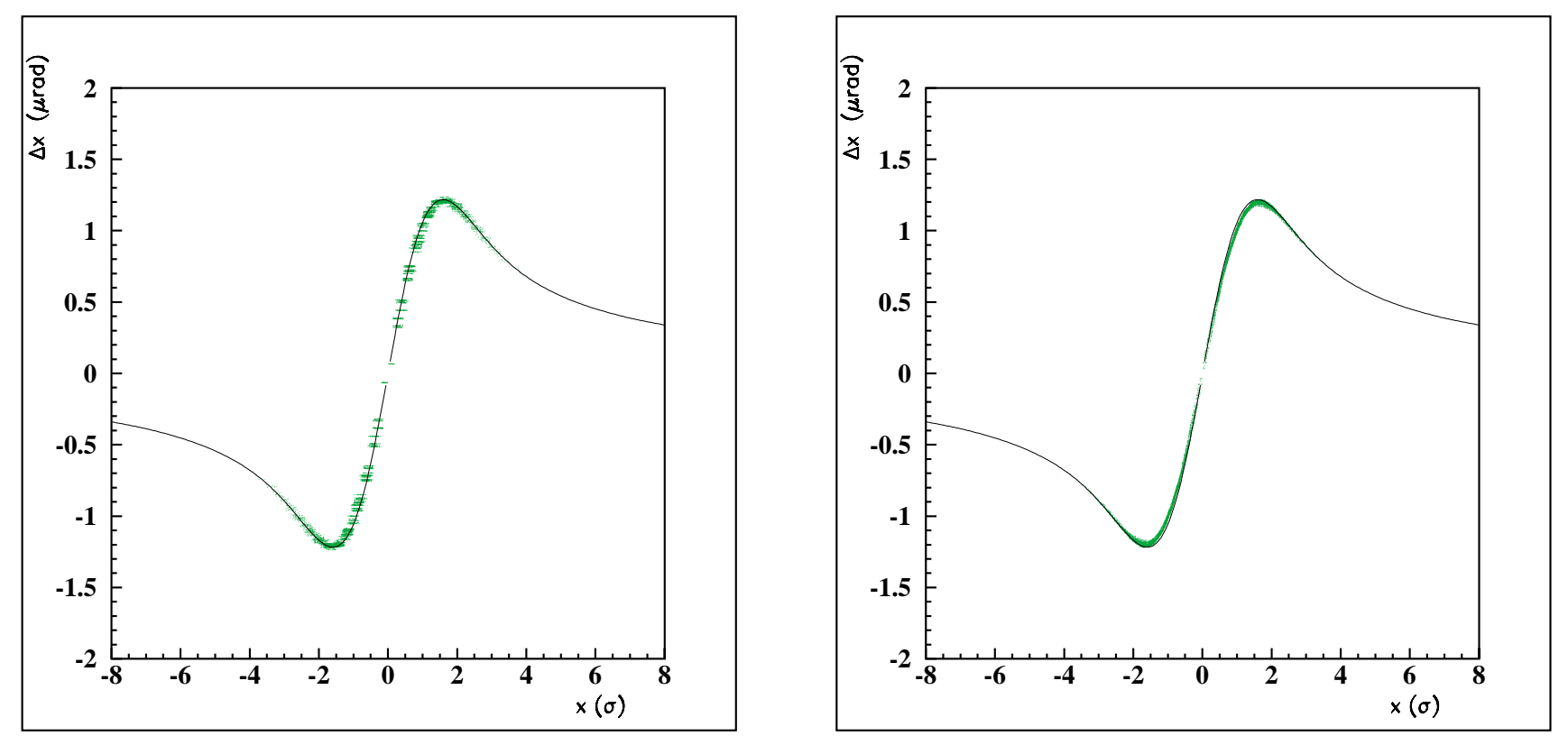

FIG. 1. (Color) Beam-beam kick as calculated with HFMM (points) and from analytical expression (solid line) for round beams with Gaussian distribution. Left: $0.25 \sigma$ grid $(81 \times 81)$ and nearest-grid-point assignment. Right: CIC assignment.

distribution at a distance $x=L_{x} \sigma_{x}$. This assumes that a closed orbit exists [11] and the bunches oscillate coherently around this orbit. At the LHC, there are about $n_{\text {par }}=16$ parasitic encounters on each side of an IP, with a minimum transverse separation of $L_{x}=7.5$ (in units of $\sigma_{x}$ ). The fractional parts of the horizontal and vertical tunes are 0.31 and 0.32, and, unlike the Large Electron Positron Collider (LEP) [13], the results are not strongly affected by dynamic beta effects. In Figs. 1 and 2 we show comparisons between the beam-beam kicks calculated with the

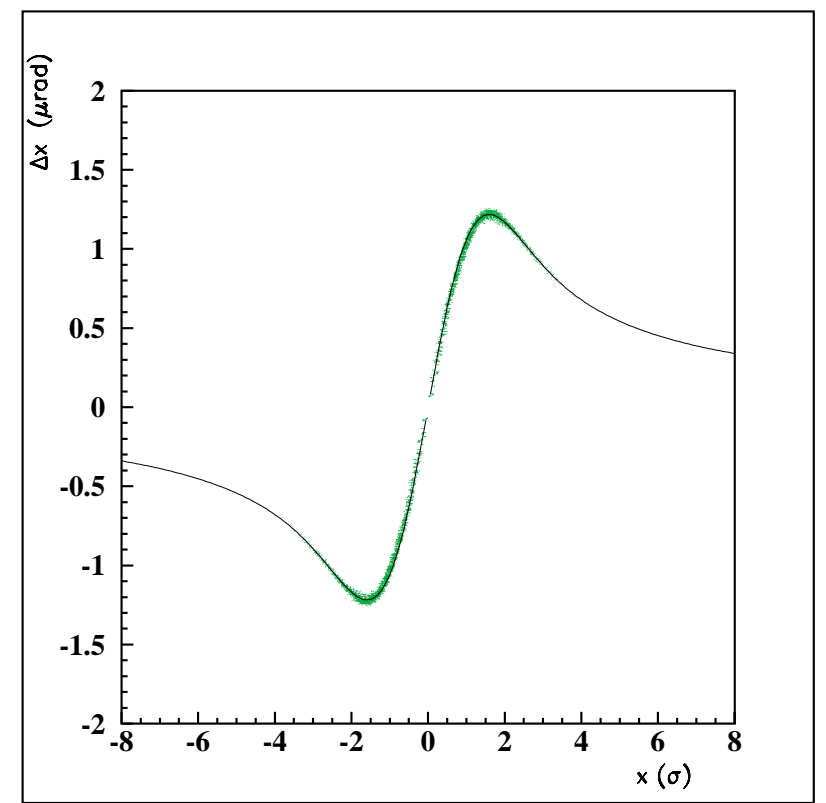

HFMM and those obtained from an analytical expression, both for the case of round, exactly Gaussian beams. In Fig. 1 we test the different methods for the charge assignment for a grid spacing of $0.25 \sigma$ with a grid of $81 \times 81$, where 81 is the number of grid points in each plane. Thus the grid for the head-on collisions covers the amplitudes between $-10 \sigma$ and $+10 \sigma$. While the nearest-grid-point assignment gives visibly discontinuous values, the force evaluated with the CIC assignment is continuous and therefore preferable.

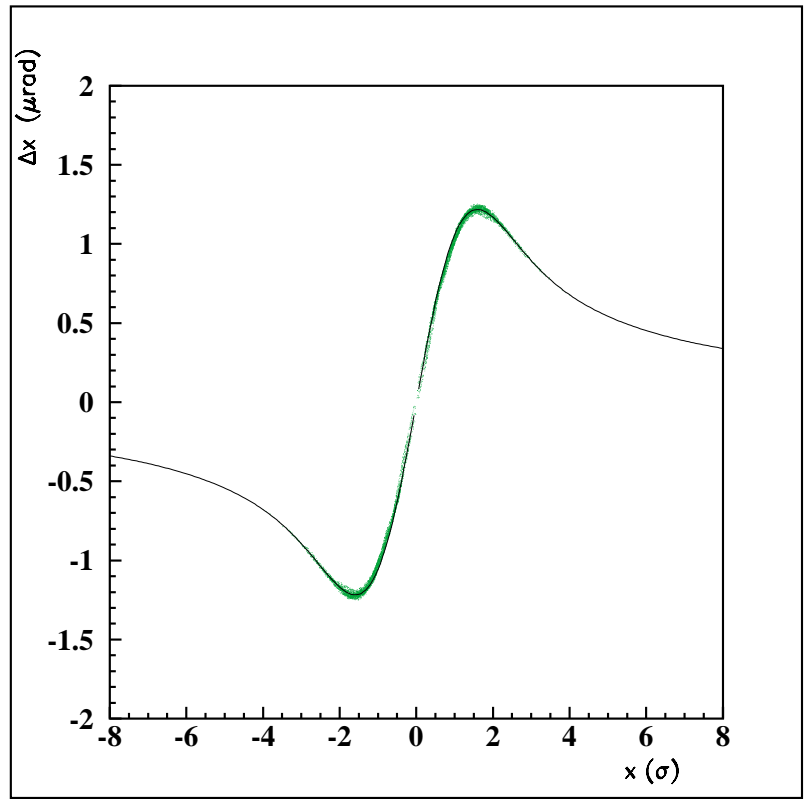

FIG. 2. (Color) Beam-beam kick as calculated with HFMM (points) and from analytical expression (solid line) for round beams with Gaussian distribution. Left: $0.10 \sigma$ grid $(201 \times 201)$ and nearest-grid-point assignment. Right: CIC assignment. 
In Fig. 2 we used a different grid spacing of $0.10 \sigma$ with a grid of $201 \times 201$ to test the obtained accuracy. The effect of the discontinuous values in the nearest-grid-point assignment is now smaller and barely visible, as one could expect. The grid size for the simulation is a compromise between precision and computing speed. A grid spacing of $0.1 \sigma$ or below gives good results. For most simulations we have therefore chosen such a spacing and the CIC charge assignment.

\section{SIMULATION RESULTS}

In this section we give quantitative results on the coherent modes for head-on as well as some first results with long-range interactions. Since the symmetry of beam parameters plays an important role for the coherent motion, we study the relevance of intensity differences as well as tune and beam size asymmetries. They are expected to make it more difficult to maintain a coherent motion and will eventually help to avoid it.

\section{A. Head-on collisions with equal betatron tunes and intensity}

First, let us consider the strong-strong case and head-on collisions of two round bunches, using the previous maps. The statistical variation in the initial distribution of particles is sufficiently large to excite the coherent modes. We start with equally strong beams; i.e., the intensity ratio $R_{I}$ between the weaker and stronger beam is 1.0. If we perform a harmonic analysis of the motion of the barycenter of one bunch, we find two coherent modes. One is located at the unperturbed tune $Q$, the other has a lower frequency. In Fig. 3 we plot the amplitude frequency spectrum. The horizontal axis gives the tune shift from the unperturbed tune $Q$ in units of $\xi$ (i.e., $w=\frac{\nu-Q}{\xi}$, for the round beam case $\xi_{x}=\xi_{y}=\xi=0.0034, Q_{x}=0.31, Q_{y}=$ 0.32). For the other beam and the other plane a similar picture is obtained. Analyzing the spectra of the distance between the centroids, i.e., the expressions $\left\langle x^{(1)}\right\rangle-\left\langle x^{(2)}\right\rangle$ and $\left\langle y^{(1)}\right\rangle-\left\langle y^{(2)}\right\rangle$, the coherent mode at the unperturbed frequency disappears. On the other hand, when we analyze the sum of the centroids $\left(\left\langle x^{(1)}\right\rangle+\left\langle x^{(2)}\right\rangle,\left\langle y^{(1)}\right\rangle+\left\langle y^{(2)}\right\rangle\right)$ the lower mode frequency disappears. We can thus identify the mode at the unperturbed frequency as the so-called $\sigma$ mode for which the centroids of the bunches oscillate in phase with equal frequencies and amplitudes. The lower frequency mode is called $\pi$ mode, and in this mode the centroids oscillate also with equal frequencies and amplitudes but in opposite phase. The motion of the bunch centroids is a superposition of these two modes.

Between the $\pi$ and the $\sigma$ mode in Fig. 3 we find the incoherent continuum. A single particle crossing the opposing beam at a distance from its axis feels a defocusing force (or focusing force in the case of oppositely charged beams such as LEP), which leads to a change in its tune.

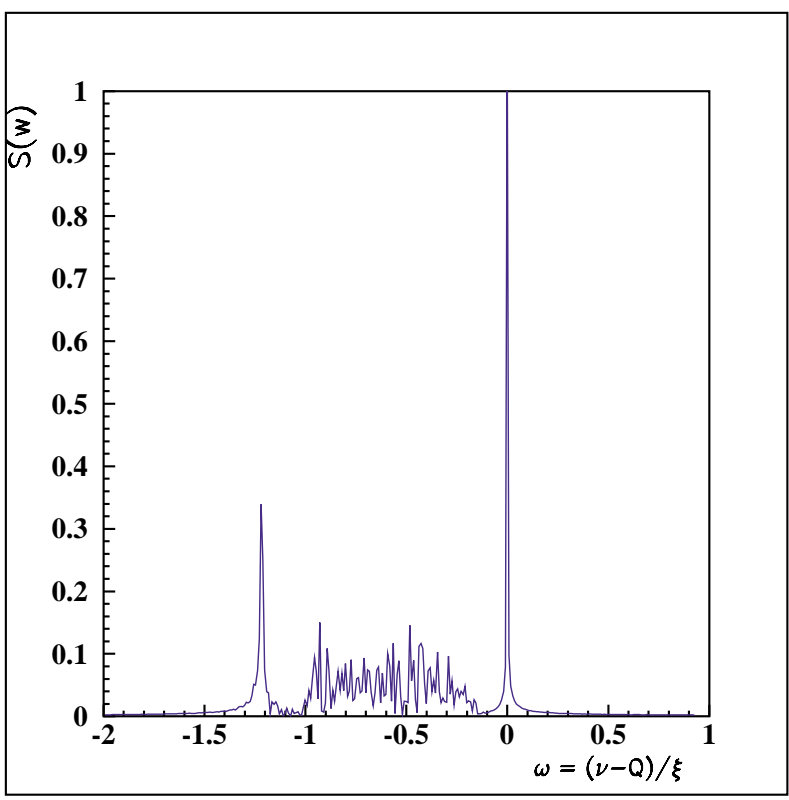

FIG. 3. (Color) Frequency spectrum of the bunch centroid motion (for $2^{17}$ turns, $N=10^{4}$ macroparticles) for round beams. The grid covers from $-10 \sigma$ to $10 \sigma$, the rest of the particles being treated as halo particles. The horizontal axis gives the tune shift from the unperturbed tune $Q$ in units of $\xi$, i.e., $w=\frac{\nu-Q}{\xi}$. The vertical axis is the corresponding amplitude. The $\pi$ and $\sigma$ oscillation modes are clearly visible.

For particles near the center of the counterrotating beam this tune shift is equal to $-\xi$. For particles farther away, the defocusing force is smaller (due to the nonlinearity of the beam-beam force) and vanishes asymptotically. This creates an incoherent tune spread which extends from 0 to $-\xi$.

In our simulations we find the $\pi$ mode at a tune shift of exactly $1.21 \pm 0.005$ in units of $\xi$ (and $\xi=0.0034$ ). The $\pi$ mode is thus shifted outside of the continuum. The shift calculated with HFMM is therefore in excellent agreement with the theoretical prediction $[1,4]$.

\section{B. Head-on collisions with equal betatron tunes and different intensity}

It was predicted [4] that for intensity ratios of 0.6 or lower, the $\pi$ mode merges with the continuum. In the soft Gaussian model this prediction cannot be tested exactly since the $\pi$ mode tune shift is underestimated $[2,3]$. In this section we can now make a more precise quantitative comparison. Figure 4 clearly confirms this prediction: the $\pi$ mode merges into the incoherent spectrum at Alexahin's ratio of 0.6 and is Landau damped. In the LHC the expected bunch-to-bunch intensity difference may be as large as $\pm 20 \%$. Although this alone will not be sufficient to recover Landau damping, together with other uncertainties (see, e.g., Sec. V D) and suggested remedies (see next section) it should simplify the damping of the modes. 

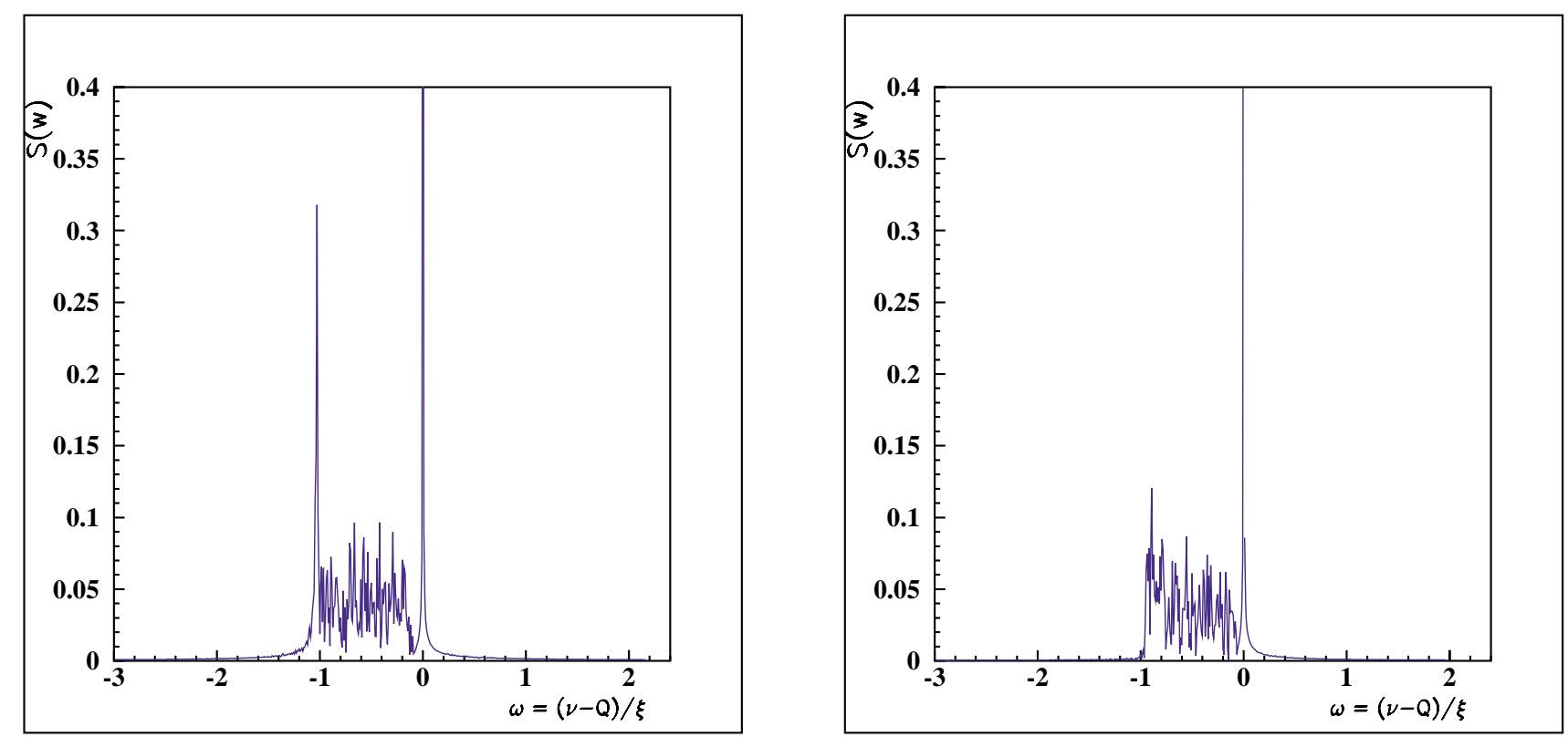

FIG. 4. (Color) Frequency spectrum of the bunch centroid motion (over $2^{17}$ turns, $N=10^{4}$ macroparticles) for round beams and intensity ratio $R_{I}=0.65$ (left) and 0.55 (right).

\section{Head-on collisions with different betatron tunes}

The first proposed remedy to avoid coherent beam-beam modes was to decouple the two beams by using different fractional tunes for their tunes [14]. This is possible in the LHC since we have two separate rings. Possible unwanted side effects of such a scheme were discussed in [15]. The sensitivity to the expected small tune differences is demonstrated here quantitatively. While the fractional

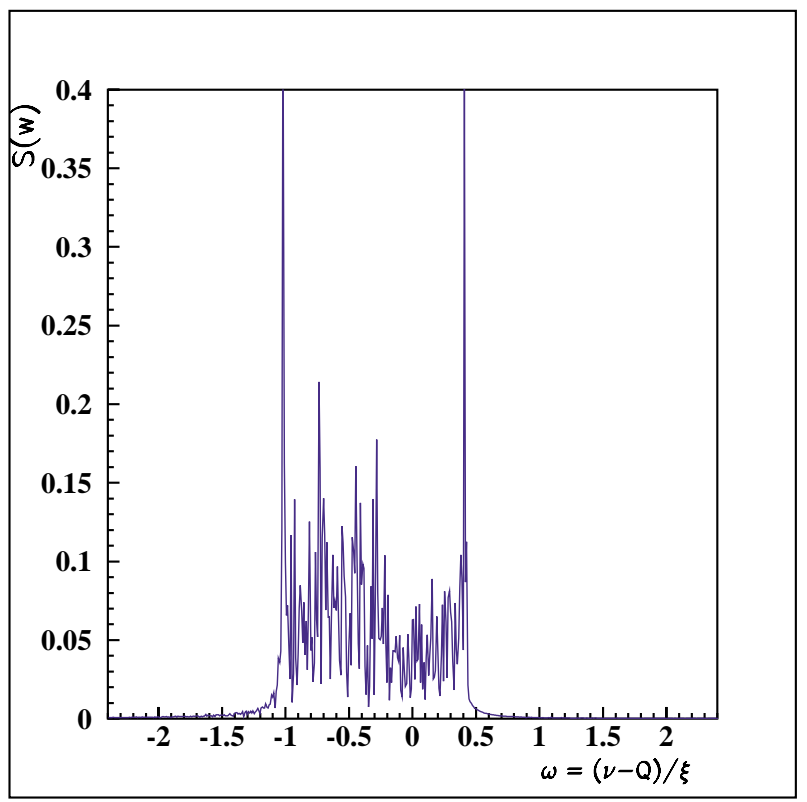

part of beam one is kept at 0.310 , the tune of the second beam is slightly varied. For a tune difference between the two beams of more than $\approx 0.7 \xi$, the $\pi$ mode disappears into the continuum, as shown in Fig. 5.

\section{Head-on collisions with different beam sizes}

Similar to an intensity imbalance, different beam sizes of the two beams can lead to loss of coherence and damped

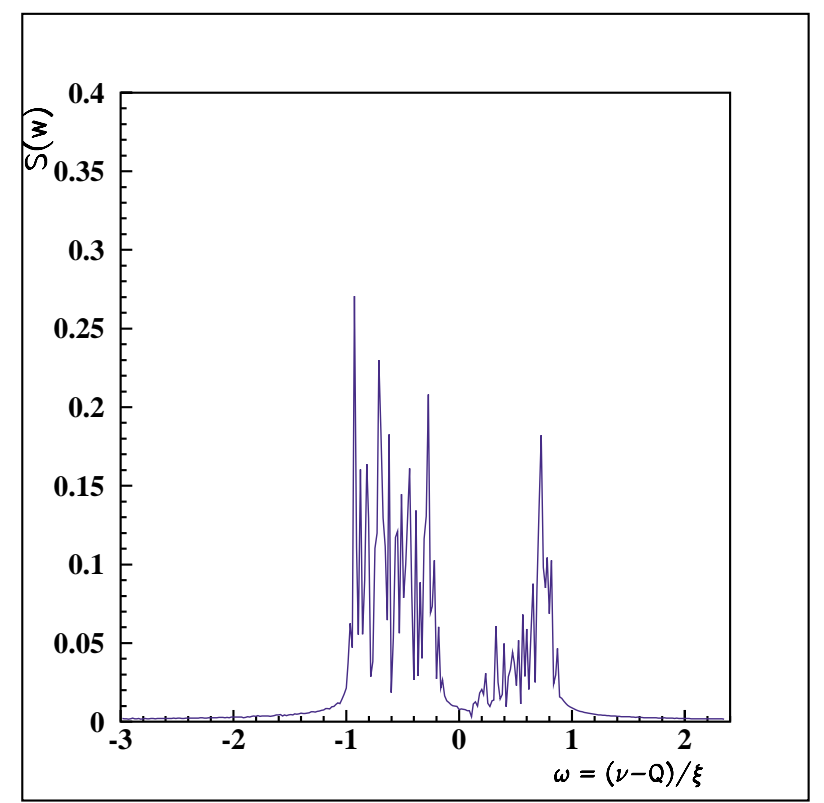

FIG. 5. (Color) Frequency spectrum of the bunch centroid motion (for $2^{17}$ turns, $N=10^{4}$ macroparticles) for round beams and different fractional tunes of the second beam: 0.312 (left) and 0.313 (right). The tune of the first beam is kept at 0.310 . 

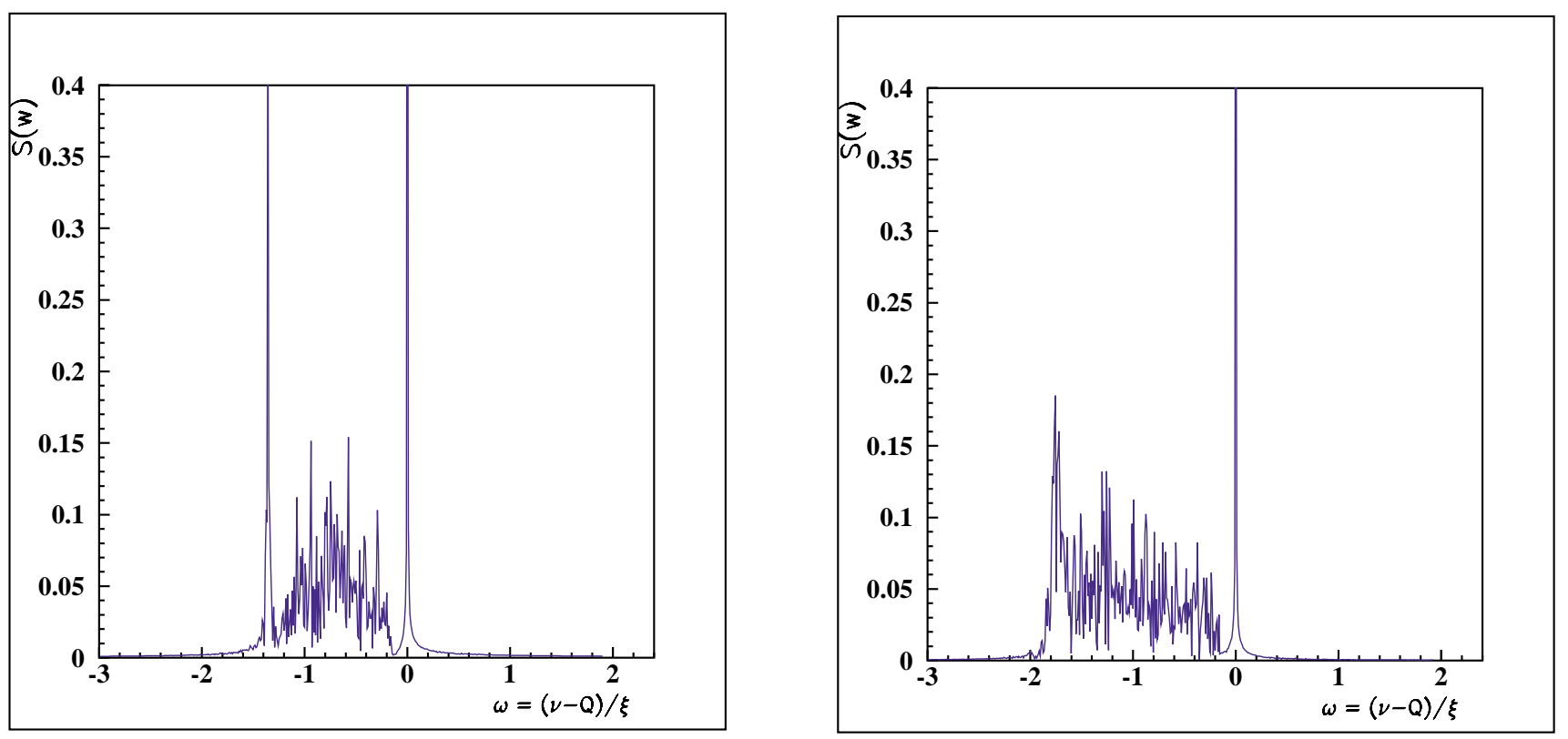

FIG. 6. (Color) Frequency spectrum of the bunch centroid motion (for $2^{17}$ turns, $N=10^{4}$ macroparticles) for round beams and size ratios $\sigma^{(2)} / \sigma^{(1)}$ of 0.90 (left) and 0.70 (right).

coherent modes. In Fig. 6 we show the spectra for beam size ratios of 0.90 and 0.70 . Since the beam size (of the second beam) is now smaller, the tune shift is slightly larger than in the original case. While for a ratio of 0.90 the $\pi$ mode is still very visible, it has merged with the incoherent spectrum for 0.70 . The mechanism is the same as for a beam intensity imbalance. At this point one can speculate whether the size imbalance can be compensated by an

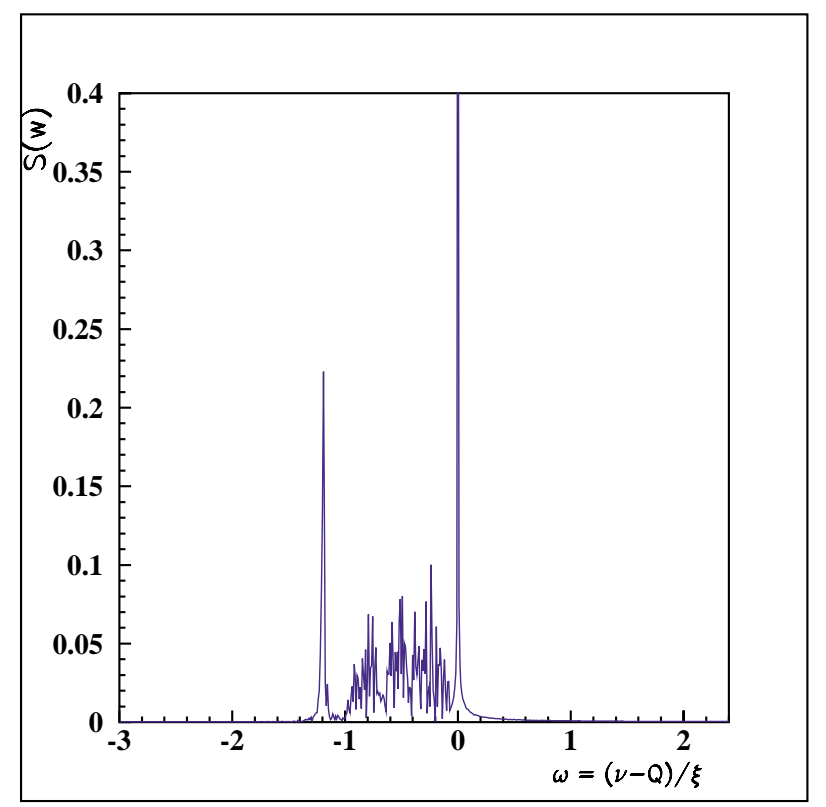

FIG. 7. (Color) Frequency spectrum of the bunch centroid motion (for $2^{17}$ turns, $N=10^{4}$ macroparticles) for round beams and size ratios $\sigma^{(2)} / \sigma^{(1)}=0.70$ and intensity ratio $R_{I}=0.5$. intensity imbalance, adjusted to give the same beam-beam tune shift parameter $\xi$. The result of such a simulation is shown in Fig. 7 with the beam radius of the second beam reduced to 0.7 , but with a smaller beam intensity $(50 \%)$. The beam-beam parameter is therefore the same. We observe a clear coherent mode again. This observation, however, is nontrivial. When the beams have different sizes and geometrical distributions, the fields seen by the two beams are rather different, although the tune shift parameter for the small amplitude particles is the same. The reason is that the larger beam experiences a very nonlinear force for particles at much smaller amplitudes than the smaller beam. Particles at larger amplitudes must therefore behave rather differently. For the single particle behavior, i.e., population of beam tails and lifetime, this is known to be of extreme importance $[16,17]$. For a coherent oscillation it is mainly the oscillation frequency that must be the same, and it is known that, for the head-on collisions studied in this example, it is mainly the core of the beam contributing to the coherent oscillation and the tune shift. The core particles always experience an almost linear force proportional to the beam-beam parameter, and this explains the observation.

Similar observations have been made in simulations of asymmetric colliders such as PEP-II [18] where the energy transparency condition was studied, i.e., where the energy asymmetry was compensated by an asymmetry of the beam currents.

\section{E. Coherent modes from long-range collisions}

Since the transverse distance between two bunches at the parasitic collision is larger than the rms beam size, the 
effects will be similar to the coherent interaction of rigid, pointlike bunches. In that case the contribution of parasitic crossings to the tune shift of coherent oscillation modes would be

$$
\Delta \nu_{\pi}=2 \times \text { (incoherent long-range tune shift) } \propto 1 / L_{x}^{2},
$$

$$
\Delta \nu_{\sigma}=0 .
$$

Moreover, the incoherent long-range tune shifts for beam separations larger than $\approx 1.5 \sigma$ have different signs for the two planes. Both the coherent and incoherent tune shifts depend on the separation, and for sufficiently large separation they scale with the inverse of the separation squared.

Most important, however, the width of the incoherent spectrum (tune spread) of long-range collisions alone depends on the separation and in the LHC is smaller than the tune spread from head-on collisions $[19,20]$. The distance of the $\pi$ mode from the edge of the incoherent spectrum is therefore rather different from the head-on case and one must expect a different behavior. In particular, the necessary measures to merge the coherent modes with the incoherent spectrum must be at least quantitatively different. In this report we have a first look at the dynamics of long-range collisions separately to demonstrate the differences. For an evaluation of the necessary operational parameters, both head-on as well as long-range collisions must be considered together as was done with the Gaussian approximation [2]. A more complete study should also include multiple bunches and interaction points and will be treated at a later stage [21].

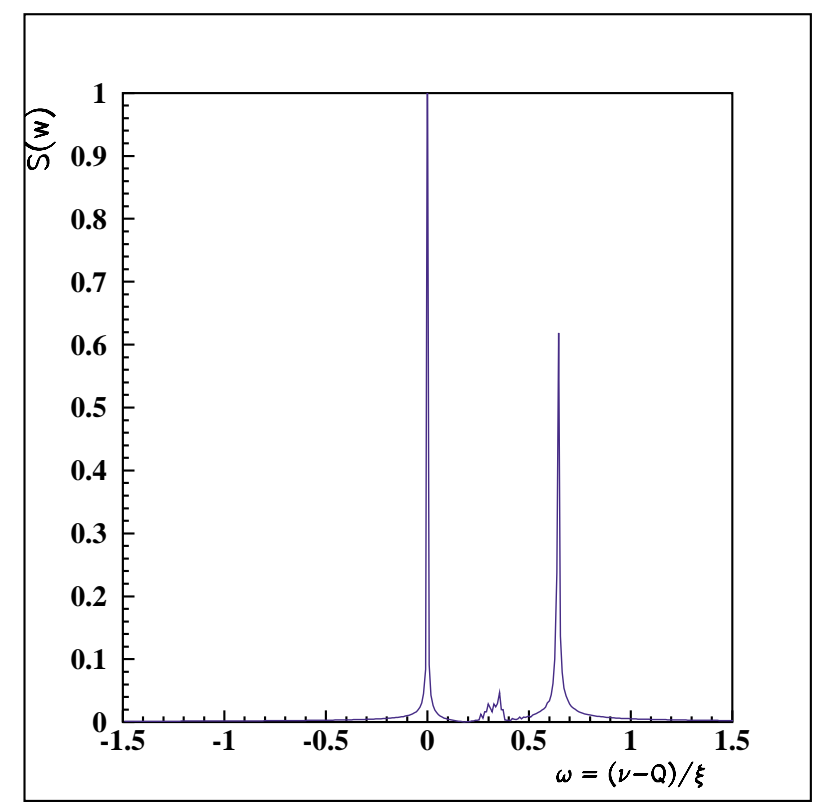

\section{F. Simulation of long-range collisions}

The simulation of coherent modes from separated beams is a good example where the HFMM can be used to great advantage. In a conventional particle-mesh method, most grid points between and around the beams are empty, and with a typical separation around $10 \sigma$ the necessary computing time becomes unacceptable. With the HFMM we have the option to either treat the opposing beam as a halo or to choose the grid large enough to cover both beams. Although at first sight the second option looks like a conventional grid method, the advantage is clear: the fields are calculated with the FMM field solver only at the grid points with charges and the saving in computing time is large. Treating the opposing beam as a real halo object usually requires more time than covering the whole area. In Fig. 8 we show the horizontal spectrum for long-range collisions with a horizontal separation $L_{x}=10.0$ (in units of $\sigma_{x}$ ). We plot it again as a function of the distance to the unperturbed tune, normalized to the head-on beam-beam tune shift $\xi$, to allow a quantitative comparison to the head-on modes. For one of the figures (left side) the particles in the opposing beam were treated as halo particles, i.e., were not covered by the grid. In the right-hand figure the grid was extended to $15 \sigma$, i.e., included both beams. Both methods give the same results; however, the computing speed is very different. The treatment as real halo is very time consuming. The real difference to a particle-mesh code then comes from the fact that only grid points with particles are treated, thus the number of computations scales as $O\left(N_{p}\right)$. The computing speed difference is about a factor of 2.5 between the two options; therefore, in all simulations we

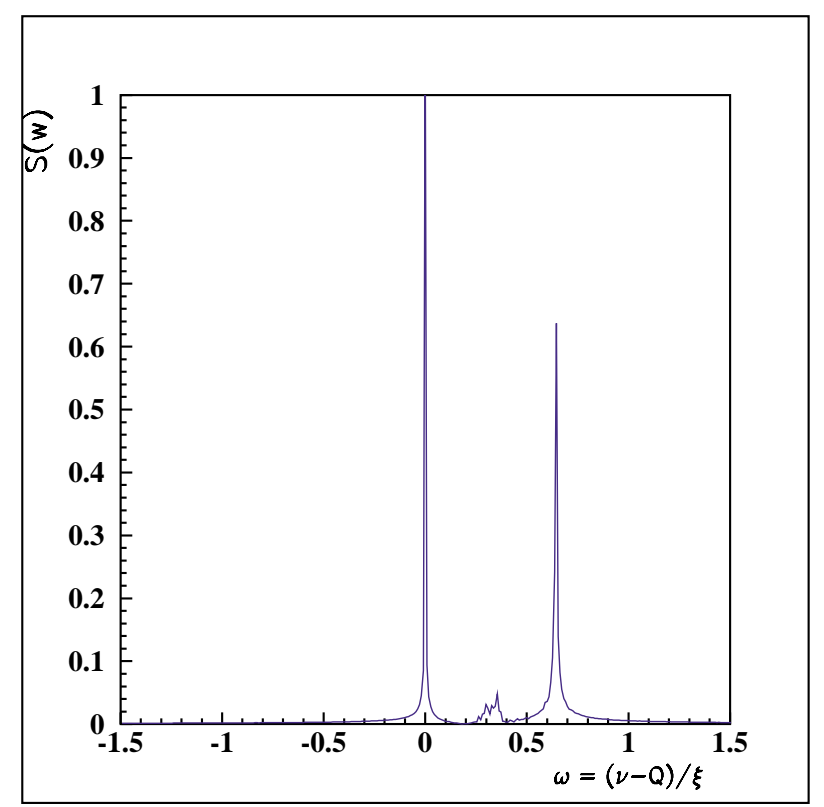

FIG. 8. (Color) Spectrum of the horizontal centroid motion for long-range collisions with horizontal separation $L_{x}=10.0$ (in units of $\left.\sigma_{x}\right)$ and no head-on collision ( $2^{15}$ turns, $N=10^{4}$ macroparticles). For the left figure the grid did not cover both separated beams; i.e., the particles in the second beam were treated as halo particles. In the right figure the grid covered both beams. 
choose the procedure to cover the whole area with a grid, including both beams.

As in the case of head-on coherent modes, we identify the $\sigma$ and $\pi$ modes easily by analyzing the sum and the difference of the barycenters separately. The peaked structure between the two modes again represents the incoherent continuum, this time arising from the long-range

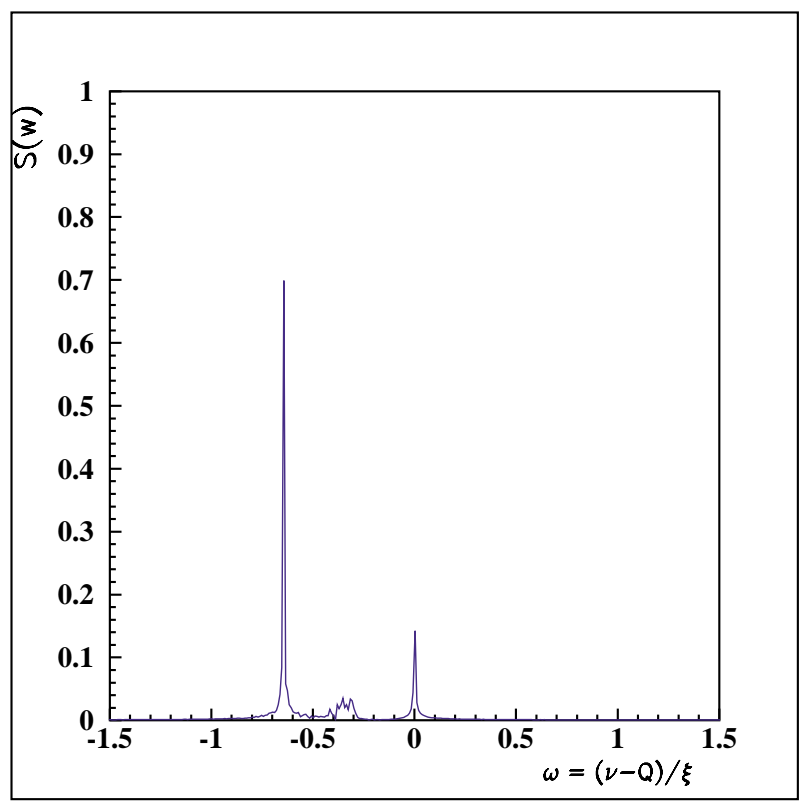

interaction. As expected, the coherent shift is 2 times larger than the shift of the incoherent spectrum.

\section{G. Long range collisions with equal tunes}

Figure 9 shows the horizontal and vertical spectra of centroid oscillations of a bunch subject to long-range

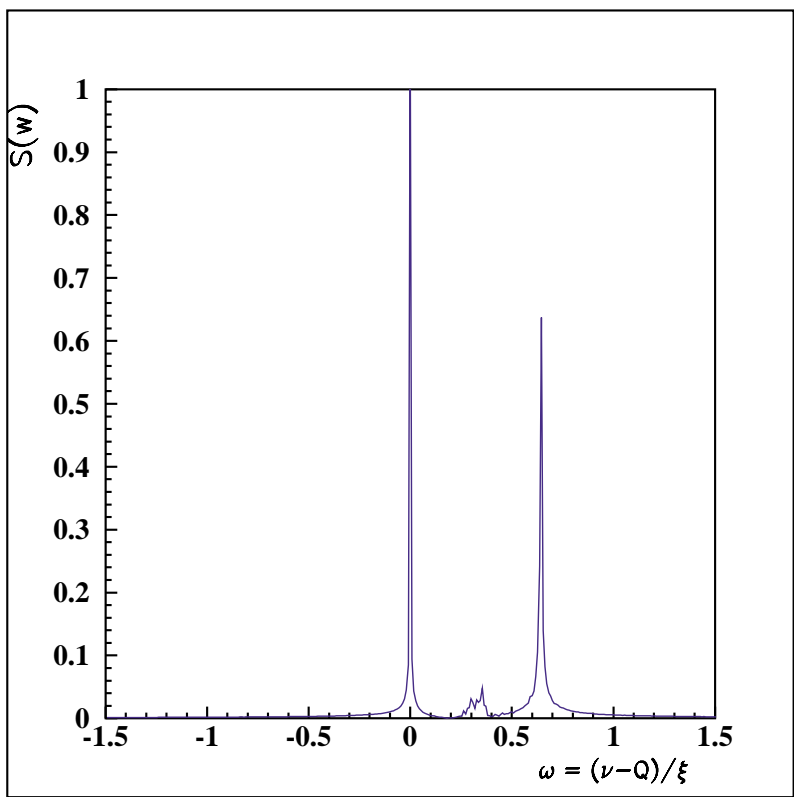

FIG. 9. (Color) Spectrum of the vertical (left) and horizontal (right) centroid motion for long-range collisions with horizontal separation $L_{x}=10.0$ (in units of $\sigma_{x}$ ) and no head-on collision ( $2^{15}$ turns, $N=10^{4}$ macroparticles). The tune shifts due to long-range collisions have opposite signs in the two transverse planes. The coherent $\pi$ mode is at twice the incoherent tune shift.
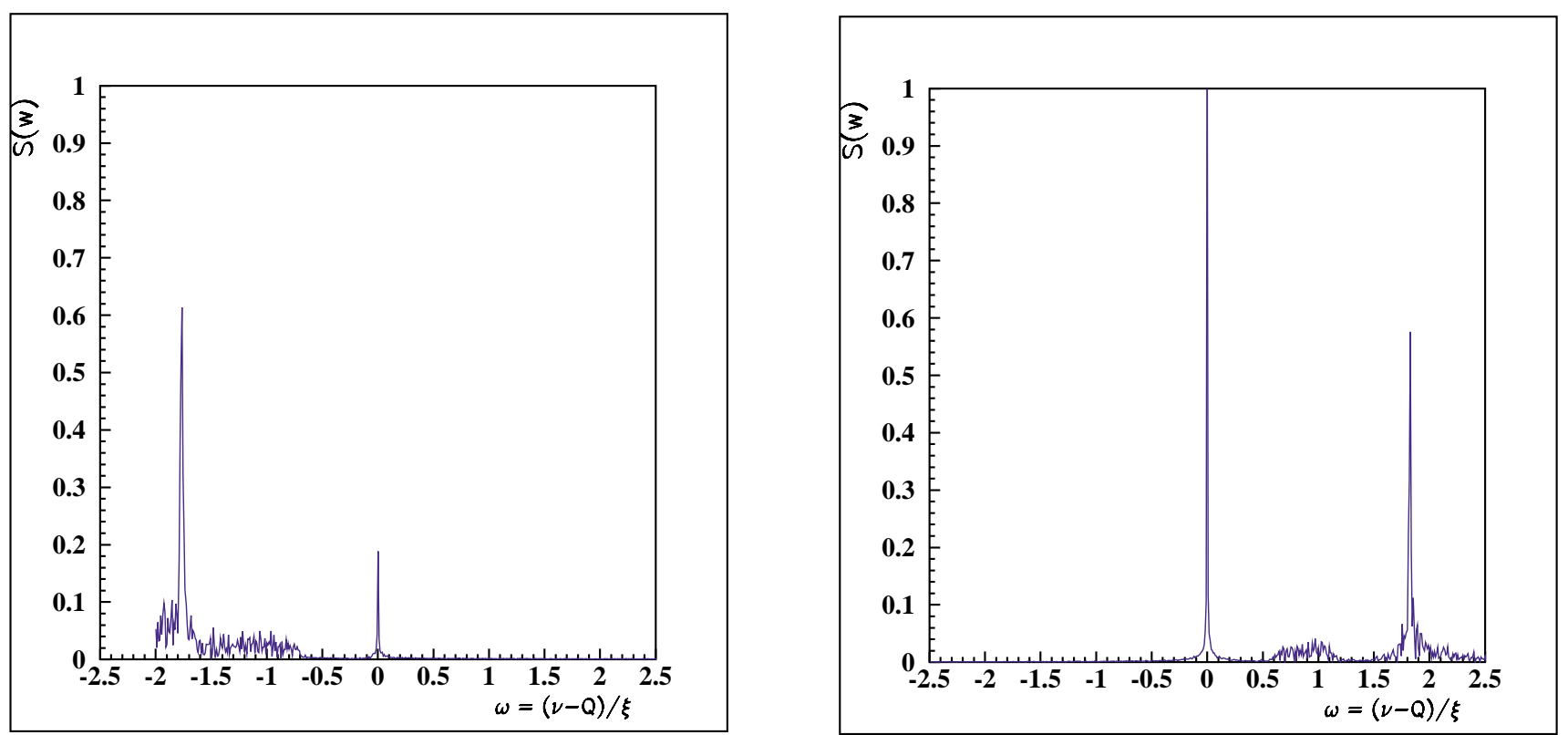

FIG. 10. (Color) Spectrum of the vertical (left) and horizontal (right) centroid motion for long-range collision with horizontal separation $L_{x}=6.0$ (in units of $\sigma_{x}$ ) and no head-on collision ( $2^{15}$ turns, $N=10^{4}$ macroparticles). The tune shifts due to long-range collisions have opposite signs in the two transverse planes. 
collisions with a horizontal separation of $L_{x}=10.0 \sigma_{x}$. To obtain realistic tune shifts, we have lumped all 32 long-range interactions of a LHC interaction region into a single collision. The optics and geometry of the interaction regions permit this simplification $[2,12]$. The horizontal axis gives the tune shift relative to the unperturbed tune $Q$ in units of the head-on beam-beam parameter $\xi: w=$ $\frac{\nu-Q}{\xi}$. In the horizontal plane, the tune shifts are positive, and the coherent dipole $\pi$ mode has twice the incoherent tune shift. In the vertical plane, the tune shifts are negative. The normalized tune shifts of the $\pi$ modes are $\left(w_{x}, w_{y}\right)=(0.645 \pm 0.005,-0.644 \pm 0.005)$. In Fig. 10 we show the results for a separation of $6.0 \sigma_{x}$ and find values of $\left(w_{x}, w_{y}\right)=(1.828 \pm 0.005,-1.762 \pm 0.005)$. Comparing Figs. 9 and 10, the larger tune shift for the smaller separation is clearly visible as well as the increased tune spread of the incoherent spectrum. Both scale with $1 / L_{x}^{2}$ as expected.

\section{CONCLUSIONS}

We implemented the HFMM technique to describe the beam-beam collision of two beams in the strong-strong regime. This allows us to study, by means of multiparticle tracking and with no approximation in the evaluation of the electromagnetic force, the coherent modes of oscillations of two colliding beams. Future improvements will extend this work to several bunches per beam and, in particular, will allow us for the first time to study details of the modes excited by long-range interactions.

\section{ACKNOWLEDGMENTS}

We would like to express our gratitude to M. Craddock (TRIUMF) for supporting this study and to M. D'Yachkov (TRIUMF) who first suggested the use of the fast multipole method for our problem.
[1] K. Yokoya and H. Koiso, Part. Accel. 27, 181 (1990).

[2] M.P. Zorzano and F. Zimmermann, CERN LHC Project Report No. 314, 1999.

[3] K. Yokoya, Phys. Rev. ST Accel. Beams 3, 124401 (2000).

[4] Y. I. Alexahin, Part. Accel. 59, 43 (1999).

[5] Y. I. Alexahin, CERN LHC Project Report No. 467, 2001.

[6] S. Krishnagopal and R. Siemann, Phys. Rev. Lett. 67, 2461 (1991).

[7] L. Greengard, The Rapid Evaluation of Potential Field in Particle Systems (MIT Press, Cambridge, MA, 1988).

[8] L. Greengard and V. Rokhlin, J. Comput. Phys. 73, 325 (1987).

[9] F. W. Jones, in Workshop on Space Charge Physics in High Intensity Hadron Rings, edited by A. U. Luccio and W. T. Weng, AIP Conf. Proc. No. 448 (AIP, New York, 1998).

[10] F. W. Jones, in Proceedings of the 1997 Particle Accelerator Conference, Vancouver, Canada (IEEE, Piscataway, NJ, 1997).

[11] H. Grote, CERN LHC Project Report No. 404, 2000.

[12] W. Herr, CERN Report No. CERN/SL/91-34 (AP), 1991.

[13] D. Brandt, W. Herr, M. Meddahi, and A. Verdier, in Proceedings of the 1999 Particle Accelerator Conference, New York (IEEE, Piscataway, NJ, 1999).

[14] A. Hofmann, in Proceedings of the LHC-99 Beam-Beam Workshop at CERN, 1999, edited by J. Poole and F. Zimmermann (CERN, Geneva, 1999).

[15] Y. I. Alexahin and M. P. Zorzano, CERN LHC Project Note No. 226, 2000 (unpublished).

[16] M. Meddahi, Ph.D. Thesis, Université Paris 7, 1991; CERN Report No. CERN SL 91-30 (BI), 1991.

[17] L. Evans, J. Gareyte, M. Meddahi, and R. Schmidt, in Proceedings of the 1989 Particle Accelerator Conference, Chicago, 1989 (IEEE, Piscataway, NJ, 1989).

[18] S. Krishnagopal, Phys. Rev. ST Accel. Beams 3, 024401 (2000).

[19] W. Herr and J. Miles, CERN LHC Project Note No. 4, 1995 (unpublished).

[20] O. Meincke and H. Grote, CERN LHC Project Note No. 161, 1998 (unpublished).

[21] W. Herr, M. P. Zorzano, and F. Jones (to be published). 